\title{
Focus on Science, Not Format: Introducing No Hassle Submissions to the Journal of Hospital Medicine
}

\author{
Samir S Shah, MD, MSCE1,2
}

'Divisions of Hospital Medicine and Infectious Diseases, Cincinnati Children's Hospital Medical Center, Cincinnati, Ohio; ${ }^{2}$ Department of Pediatrics, University of Cincinnati College of Medicine, Cincinnati, Ohio.

(a) he Journal of Hospital Medicine ${ }^{\circledR}$ is committed to continually improving the author experience. Our goal is to allow authors to focus more time on communicating their message and less time on navigating the submission and publication process. We commit to three initial areas of emphasis: (1) Make it easy for authors to submit their work; (2) Make timely disposition decisions; and (3) Facilitate dissemination of work that we publish.

We are pleased to introduce a new "No hassle" process for initial original research and brief report manuscript submissions. There is no universally followed format for manuscript submission to medical journals. ${ }^{1-3}$ As a result, authors spend considerable time reformatting manuscripts for submission to meet each journal's unique requirements before knowing whether or not their manuscript will be accepted for publication-or even sent for peer review. To streamline the submission process and eliminate unnecessary and burdensome reformatting, we have eased formatting requirements for initial manuscript submissions. We will even accept all manuscript elements in a single PDF (portable document format) file in another journal's format if your manuscript was submitted elsewhere first but not accepted for publication. Tables and figures can be included in the single document or uploaded separately, depending on your preference. Of course, common elements necessary to assess a manuscript, including declaration of funding sources and conflicts of interest, are required on the title page. ${ }^{1}$ Journal-specific formatting and signed disclosure and copyright forms will be deferred until a revision request.

We also seek to make timely decisions. Our rapid turnaround allows authors to submit elsewhere expeditiously if not accepted by the Journal of Hospital Medicine. We reject approximately $50 \%$ of original research and brief report manuscripts without formal peer review. The rationale for this approach is two-fold. We want to be respectful of how we engage our peer

Corresponding Author: Samir S. Shah, MD, MSCE. E-mail: Samir.Shah@cchmc org; Telephone: 513-636-6222; Twitter: @SamirShahMD

Published online first April 17, 2019

Received: April 3, 2019; Accepted: April 3, 2019

(C) 2019 Society of Hospital Medicine DOI 10.12788/jhm.3217 reviewers and we would rather not have them spend time reviewing manuscripts that we are unlikely to publish. We also want to be respectful of our authors' time. If we are unlikely to publish a manuscript based on lower priority scores assigned by the Editor-in-Chief and other journal editors, we prefer to return the manuscript to authors for timely submission elsewhere. Our average time from submission to rejection without formal peer review is 1.3 days (median, $<1$ day). If we send a manuscript out for peer review, our time from submission to first decision is 23 days. Further, if we request a manuscript revision, we sincerely hope to publish the manuscript. Thus, most manuscripts for which we request a revision are ultimately accepted for publication. We are also tracking how quickly we can publish accepted manuscripts with a goal of 120 or fewer days from submission to publication and 60 or fewer days from acceptance to publication.

We highlight our published research in many ways to facilitate dissemination. We promote articles through formal press releases, tweets, visual abstracts, and, more recently, graphic medicine abstracts or comics. Select articles are discussed through our online journal club (\#JHMChat). ${ }^{4}$ Other synergistic methods of dissemination are being planned and we'll share these ideas with you in the coming year.

We are grateful to receive a large number of submissions and are honored that authors view the Journal of Hospital Medicine as an important venue to showcase their work. We continually strive to improve the author experience and welcome your input.

\section{References}

1. International Committee of Medical Journal Editors. Recommendations for the conduct, reporting, editing, and publication of scholarly work in medical journals. Updated December 2018. www.icmje.org/recommendations/ browse/. Accessed April 2, 2019.

2. Schriger DL, Arora S, Altman DG. The content of medical journal instructions for authors. Ann Emerg Med. 2006;48(6):743-749. doi: 10.1016/j. annemergmed.2006.03.028

3. Barron JP. The uniform requirements for manuscripts submitted to biomedical journals recommended by the International Committee of Medical Journal Editors. Chest. 2006;129(4):1098-1099. doi: 10.1378/chest.129.4.1098.

4. Wray $\mathrm{CM}$, Auerbach $\mathrm{AD}$, Arora VM. The adoption of an online journal club to improve research dissemination and social media engagement among hospitalists. J Hosp Med. 2018;13(11):764-769. doi: 10.12788/jhm.2987. 